\title{
Simulation on VFTO by Phase-Selection Technology in Low Voltage Xiaoliang Ying ${ }^{1}$ \\ ${ }^{1}$ School of Electrical and Electric Engineering, North China Electric Power University, Beijing, 102206, China
}

\section{Keywords: Phase-Selection; VFTO; Low-Voltage; Disconnnector}

\begin{abstract}
Disconnector(DS) operations in GIS may generate very fasttransient overvoltage (VFTO), which is characterized by its high peak value, short rise time, and high frequencies. However,most studies on VFTO are based on statistical data, which cannot show the impact of different phases to VFTO.In this paper, we use a relay with MCU as the Phase-Selection controllerto closeto the $220 \mathrm{~V}$ power supplyin order to simulate VFTO in high-voltage substations, and acquired transientvoltage waveform of each phase. After analyzing the measured data, some important characteristics of switching fields were obtained. The lower the absolute value of the instantaneous power supply voltage is, the smaller VFTO is. When the absolute value of the instantaneous power supply is same, the amplitude of negative polarity VFTO is of about $20 \%$ smaller than the positive polarity in average, the lower the absolute value of the voltage is, the smaller the gap is. These characteristics are useful for theoretical analysis and practical engineering analysis inpower substation.If Phase-Selection controller can be used on disconnnector, then try to close at 0 degrees to left or 180 degreesto right, the offset phase is determined by controller's phase error.
\end{abstract}

\section{Introduction}

With the increase of the power system voltage level,gas insulated substation(GIS) has been widely used. The very fast transient overvoltage (VFTO) caused by operations ofdisconnectors (DS), hastriggered off more and more accidents. Switching no-load short bus by DS is the main source of VFTO. VFTO is characterized by its high peak value, short rise time, and high frequencies ${ }^{[1-5]}$, and is one of the keythreats to the insulation of high-voltage substations.

Hitherto, most studies based on statistical data, which cannot show the impact of different phases to VFTO.In this paper, a relay with MCU is used asthe Phase-Selection controller. And we use it to control aone meter long metal tube to close to the $220 \mathrm{~V}$ power supply in order to simulate high-voltage substations closingno-load short bus. The characteristics of VFTO waveform generated by operating relay in different phase are analyzed in this paper by analysing their amplitude, rising edge. Fast Fourier Transform is also used to analyze the frequencies contained in the VFTO waveform.In the end,the preventivemeasures of VFTO byoperation ofdisconnectorare discussed by analyzing the characteristics of operation ofdisconnector.

\section{Experiment Equipment Introduction}

As shown in Fig.1,power supply is lowered by step-down transformer, and then inputs to MCU with filtering and reshaping. MCU outputs a signal after it delays a specific time determined by closing phase. The signal is amplified by driving device and drives relay to close to the $220 \mathrm{~V}$ power supply. 


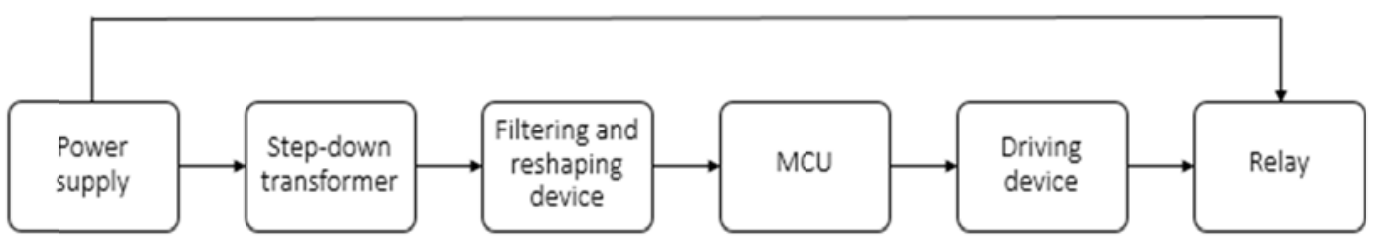

Figure1 Diagram of experiment

\section{Transient Voltage Characteristics and Analysis Generated by Relay's Operation}

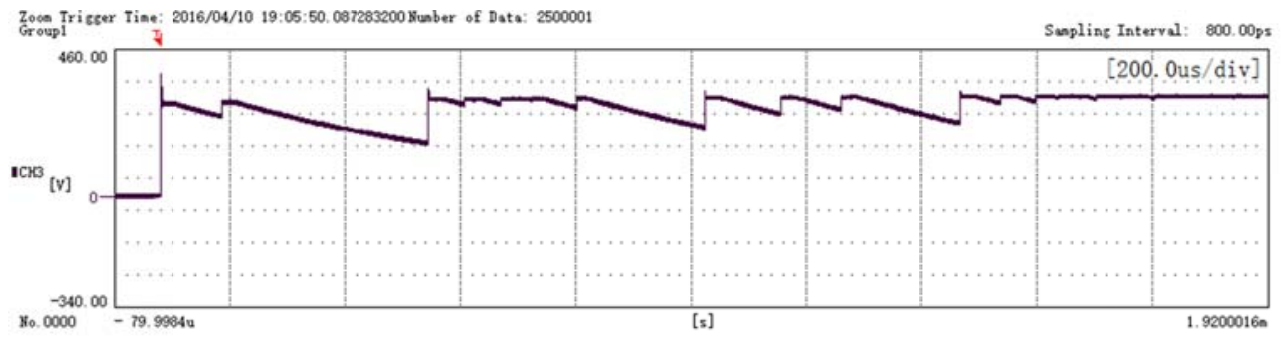

Fig 2 Waveform of transient voltagegenerated by relay closing no-load short bus

As shown in Fig.2, a dozen breakdowns occur in the closingprocess of relay. Themaximum VFTO appears in the first breakdown, the entire processlasts1 2ms.After each breakdown, the bus voltage oscillatesdamping to the power supply voltage. Because the current is too small, the arc cannot be maintained and restore insulation. The bus voltage decay exponentially with time, until the voltage between contact terminals reaches the breakdown voltage, and thenbreakdown happens again.

We can see that afterbreakdown under high voltage,there will be several breakdowns underlowvoltage.Because in tens of microseconds after breakdownunderhighvoltage, the conducting channel's temperature is very high, and it will leave a lot of ions, which makes thecontact terminals'breakdown voltage drop, and easier to breakdown.

And the voltage of breakdown in high voltage getting smaller and smaller as time increases. Becausethe contact terminals' distance is getting smaller and smaller as time increases, the breakdown voltage drops.

\section{Analysis of Transient Voltage in Different Phase}

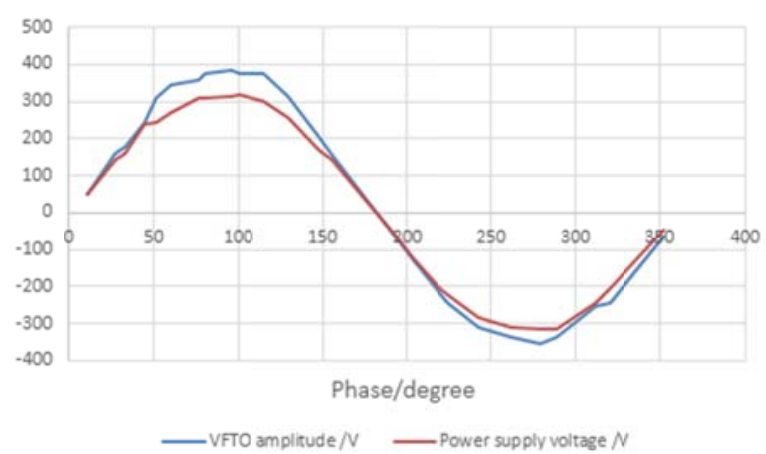

Fig 3 VFTO amplitude in different phase 


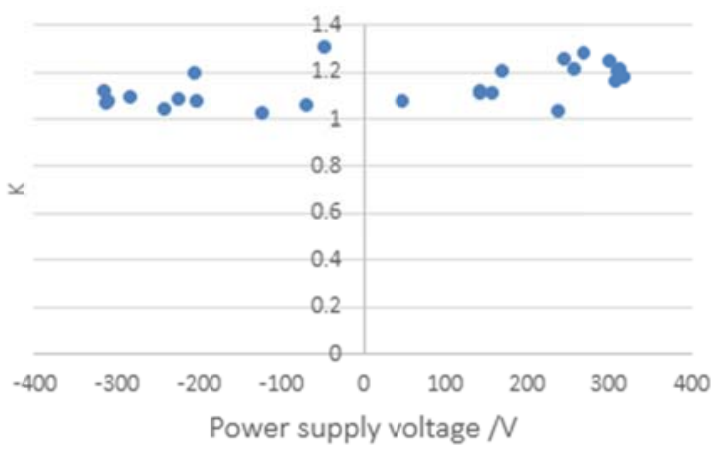

Fig4 ratio k in differentinstantaneous value ofpower supplyvoltage

As shown in Fig.3 and Fig.4, the ratio k of VFTO amplitude and instantaneous value of power supply voltage is very small,which is between $1.02 \sim 1.3$. Whenthe instantaneous value of power supply voltage is positive polarity, the higher instantaneous value of the supply voltage, kbigger; and whenthe instantaneous value of power supply voltage is negative polarity,there is no significant regularity. The $\mathrm{k}$ in negative power supplyvoltageis significantly smaller than it in positive, which is about $20 \%$ smaller. The lower the absolute value of the voltage is, the smaller the gap is.And the oscillation in each phase is unipolar, the transition process generated by single arc lasts about $0.2 \mu \mathrm{s}$.

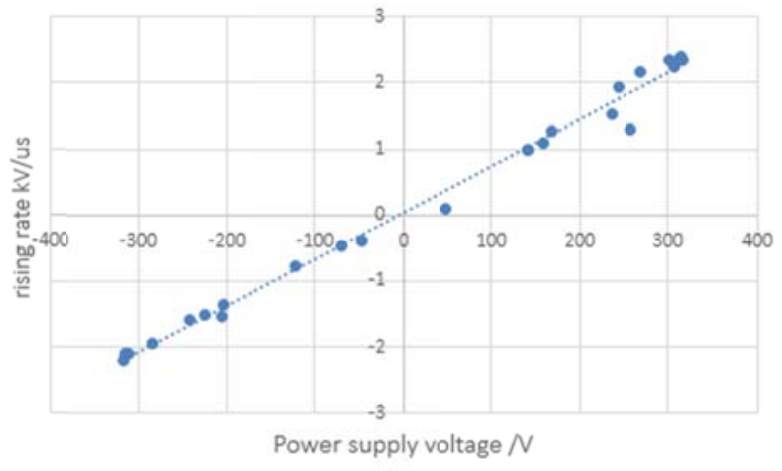

Fig 5 Voltage rising rate in different instantaneous value of power supplyvoltage

As shown in Fig.5, Voltage rising rate and instantaneous value of power supplyvoltageare approximately linear. When the supply voltage reaches the peak, the rising rate also reaches a peak of about $2.38 \mathrm{kV} / \mu \mathrm{s}$.

\section{Analysis of a Single Breakdown transient Voltage Generated by Relay's Operation}

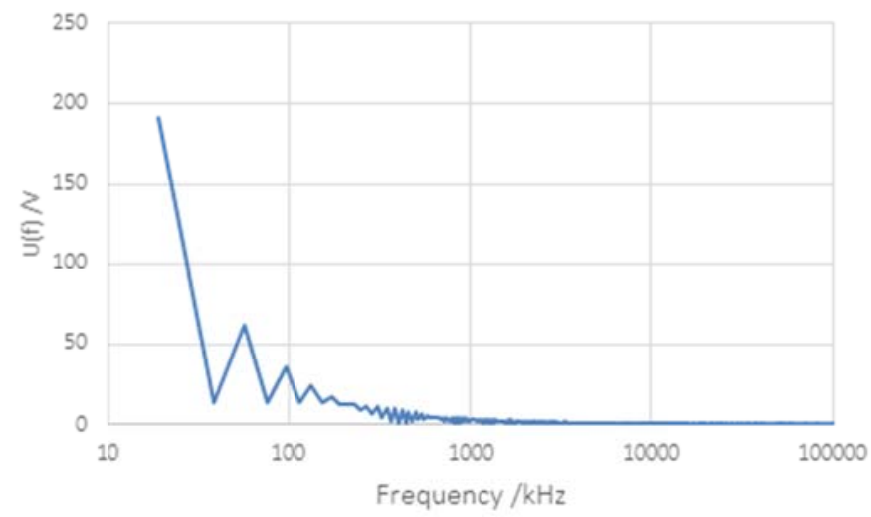

Fig 6 Spectrogram of transient voltage generated by relay closing no-load short bus

Fast Fourier Transform is used to analyze the frequencies contained in the VFTO waveform,Fig.6is a spectrogram of transient voltage generated by relay closing no-load short bus. This measurement's sampling rate $12.5 \mathrm{Ms} / \mathrm{s}$, the length of sampling time is $1 \mathrm{~ms}$.As can be seen, 
the amplitude of the DC component and low frequency components are large,theamplitude of the other components is small. Componentsgreater than about $200 \mathrm{kHz}$ are even smaller.

\section{Error Analysis of Experimental Device's Closing Phase}

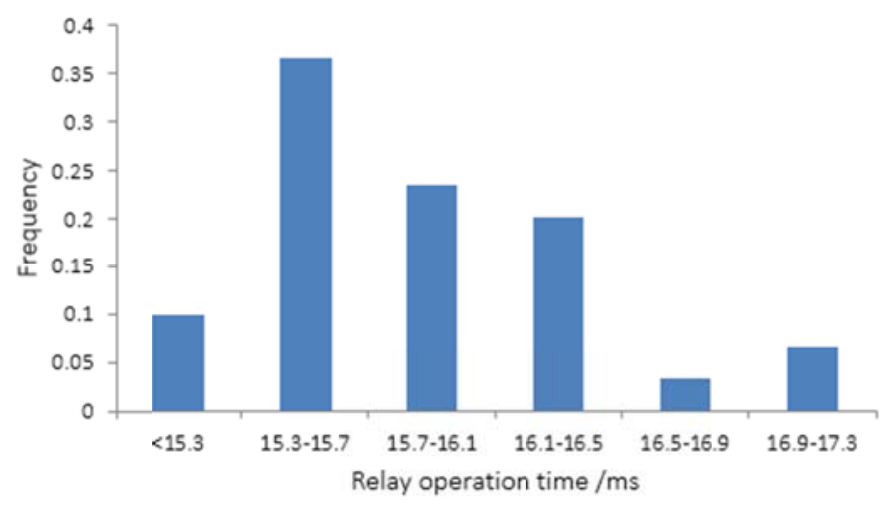

Fig 7 Relay's closing time frequency distribution histogram

(1) By measuring the time delay from the receiving of the closing signal tothe full close ofrelay contact terminals, we get a frequency distribution histogram as showed in Fig.7.By analyzing the data available, we can get the average delay time of relay closing is $15.88 \mathrm{~ms}$, variance $0.27 \mathrm{~ms}$.

(2) As can be seen from the waveform diagram, the contact terminals breakdown $1-2 \mathrm{~ms}$ earlier before fully closed, resulting in the closing phase error increases.

(3) Phase error caused by grid frequency fluctuation.

(4) MCU delay is less than $1 \mu \mathrm{s}$, which is negligible.

\section{Conclusion}

The lower the absolute value of the instantaneous power supply voltage is, the smallerVFTOis.

When the absolute value of the instantaneous power supply is same, the amplitude of negative polarityVFTO is of about $20 \%$ smaller than the positive polarity in average. The lower the absolute value of the voltage is, the smaller the gap is.

Voltage rising rate and instantaneous value of power supplyvoltageare approximately linear.

Frequency components of transient voltagegenerated by relay closing no-load short bus, are concentrated in less than $200 \mathrm{kHz}$.

So when switching device is to select phase to close in order to prevent VFTO, try to close at 0 degrees and 180 degrees. If phase cannot precisely determined, then try to close at 0 degreesto left or 180 degreesto rightso as to minimize VFTO, the offset phase is determined by controller's phase error.

With the development of disconnnector, the closing time is getting smaller. But in high-voltage substation, closing phase error increases as breakdown distance increases. So if Phase-Selection controller can be used on disconnnector, which means phase error is much less than 90 degrees, then the former conclusion can be used.

\section{References}

[1] LU Bin-xian, WANG Ze-zhong, LI Cheng-rong, et al. Measurements and research of switching operation transientelectric field in $500 \mathrm{kv}$ substations [J]. Proceedingsof the CSEE. 2004,24(4):133-138. 
[2] Lu Tiebing, Cui Xiang Numerical analysis of wave processes for bus withoutload in substations using FTDT [J]. Proceedingsof the CSEE, 2000, 20(6): 39-42.

[3] ZHONG Xiao-Quan, ZHOU Li-Xing, LIU Tao, et al. Research on Protection Measure for VFTO of 1000 kV GIS Substation [J]. Insulators and Surge Arresters. 2013, (6):119-123.

[4] HAN Bin, LIN Jiming, CHEN Weijiang, et al. Analysis on the Influence of the Switching Speed of Disconnector on Very Fast Transient Overvoltage [J]. Proceedingsof the CSEE. 2011,31(31):12-17.

[5] LIU Qin. Very Fast Transient Overvoltage Caused by Different Switching Modes of Disconnector [J]. High Voltage Apparatus. 2011,47(4):17-22. 